\title{
Business Models Innovation for Sustainable Urban Mobility in Small and Medium-Sized European Cities
}

\author{
Francesco BELLINI \\ Sapienza University of Rome/Eurokleis srl, Rome, Italy \\ francesco.bellini@uniroma1.it \\ Iana DULSKAIA \\ Eurokleis srl, Rome, Italy \\ iana.dulskaia@eurokleis.com \\ Marco SAVASTANO \\ Sapienza University of Rome, Rome, Italy \\ marco.savastano@uniroma1.it \\ Fabrizio D'Ascenzo \\ Sapienza University of Rome, Rome, Italy \\ fabrizio.dascenzo@uniroma1.it
}

\begin{abstract}
Bad air conditions, limitless traffic, overloaded parking spaces are just some of the modern mobility problems that strike cities on a daily basis. The European Commission strives to address these issues by providing measures to improve the urban mobility situation in the small and medium-sized cities. The SUITS project (Supporting Urban Integrated Transport Systems: Transferable Tools for Authorities), which is financed under the umbrella of the HORIZON 2020 programme, aims at increasing the capacity of local authorities in Small and Medium (S-M) cities through the development and implementation of sustainable, integrated and accessible transport strategies and technologies.

This paper aims to analyse and classify the innovative business models in the urban mobility sector that are aimed at increasing the capacity building of $S$ - $M$ cities' transportation authorities through the implementation of new mobility solutions. It describes the innovative mobility business identifying the most successful models of service and partnerships in the sector.
\end{abstract}

Keywords: Business Model Innovation, Small and Medium-Sized Cities, Mobility, Transportation, Digital Transformation, Sharing Economy, Horizon2020

\section{Introduction}

The capacity of developing or reshaping organisational business models requires organizational know-how and tools. Only a well-developed business strategy enables the transportation and mobility organisations to receive investments, and well-chosen partners may only reinforce the chance of success.

Bad air conditions, limitless traffic, overloaded parking spaces just some of the modern mobility problems that strike cities on a daily basis (European Commission, 2017).

A changing mobility paradigm that properly tackles today's challenges and accommodates current and emerging societal trends will clearly require research into new mobility scenarios, technological innovations, additional mobility services and solutions (Banister, 2008) as well as new partnership schemes. Over $70 \%$ of the EU population lives 
in cities (including small and medium-sized cities) and accounts for approximately $85 \%$ of the Union's GDP1 1 . The present mobility situation has created unsustainable conditions for living: severe congestion, poor air quality, noise emissions as well as a high level of C02. The increase of private vehicles brought to increased urban sprawl and commuting, however, the expansion of public transport networks has not reached the same development level (Frumkin, 2016).

European cities have common challenges that regard the enhancement of urban mobility, accessibility improvement, and creation of high quality and sustainable transport systems while reducing congestion, pollution and accidents.

Big European cities are well-known for their critical urban mobility situation, while S-M cities are left behind with respect to basic services and lack the necessary institutional capacity to manage their rapidly growing populations, and as a consequence the mobility situation (Cohen, 2006; Bell, 2006).

The European Commission has identified this problem and is searching to provide measures to solve the mobility issues in the S-M cities. For instance, the SUITS project (Supporting Urban Integrated Transport Systems: Transferable Tools for Authorities) ${ }^{2}$, financed under the umbrella of the HORIZON 2020 programme aims at increasing the capacity of local authorities in S-M cities to develop and implement sustainable, integrated and accessible transport strategies and technologies.

The SUITS project targets local authorities and stakeholders in S-M cities who are in charge of the decision-making process for creating new mobility businesses or improvements of the existing ones, developing new partnerships or preparing the projects for the investment.

Following these objectives, this study aims to contribute to the scientific literature as well as to provide the examples of innovative business models and partnerships in urban mobility to increase the capacity building of S-M cities. The innovation of this paper is contained in the provision of examples of the modern mobility trends, identifying the most successful mobility services and their business models in the mobility sector as well as innovative forms of partnerships identified during the research.

To this purpose, our research question emerged as follows:

- RQ: What are the key characteristics of the innovative business models that allow the improvement of the urban mobility situation in small and medium-sized European cities?

In order to answer our research question, this study analyses the main literature and trends on innovative business models and partnerships that can be developed in the mobility sector of S-M cities in order to improve the urban services in these settings.

This paper presents the first investigation regarding new business models for sustainable urban mobility projects in S-M-sized European cities. More profound studies in this area are foreseen in future works.

\footnotetext{
${ }^{1}$ European commission. Urban Mobility Package - https://ec.europa.eu/transport/themes/urban/urban_mobility/ump_en

${ }^{2}$ https://ec.europa.eu/inea/en/horizon-2020/projects/H2020-Transport/Urban-Mobility/SUITS
} 


\section{Literature Review \\ Urban Mobility Trends}

The current economic scenario shows that people entering the global middle class want to buy cars: automobile sales are predicted to increase from about 70 million a year in 2010 to 125 million by 2025, more than half being foreseen to be purchased in cities. Some automotive analysts predicted that the 1.2 billion strong global car fleet of today could double by 2030 (Dargay, 2007). The existing urban infrastructure cannot support such number of vehicles on the road. Congestion has already reached unbearable conditions and can cause such problems as time waste, wasted fuel, and increased cost of doing business ${ }^{3}$.

Despite these worrying facts, the transport and mobility community are trying to find solutions to the current situation. Different innovative approaches appear to solve transport problems. Many of them are due to the rapidly evolving technologies and digitalization (Benevolo et al., 2016). The technological revolution has changed the way the mobility and transport sector operate. New business models have emerged, changing the way the transport organisations run their businesses, revolutionizing the way of providing services and introducing new value propositions and types of partnerships (Bellini \& D'Ascenzo, 2019).

An example of an innovative way of improving the urban journeys is new "multimodal" services that facilitate everyday journeys combining walking, cars, buses, bikes, etc. (Spickermann et al., 2014) as well as shared transportation services and Mobility as a Service. These services not only enable the innovation of the way travellers move but also help to solve the main mobility problems and the technological development plays an important role.

In addition, the European market of Intelligent Transport Systems (ITS) is growing fast. According to a Swedish study, the market value of ITS in public transport vehicles such as buses and trams is expected to rise from €1.03bn in 2014 to $€ 1.46 \mathrm{bn}$ by 2019 . Developing apps and digital platforms-based services for smartphones or other mobile devices is a fast emerging economic sector especially regarding the urban mobility, at the base of the sharing-mobility models.

Table 1 provides the insight from the ongoing shifting paradigm in the urban mobility transportation from the traditional way towards new solutions.

Table 1. Traditional mobility schemes vs new mobility trends

\begin{tabular}{ll}
\hline Traditional mobility strategy & New mobility solutions \\
\hline $\begin{array}{l}\text { Individual car ownership as a } \\
\text { main form of transport. }\end{array}$ & $\begin{array}{l}\text { Individual car ownership as one form of } \\
\text { multimodal, on-demand and shared } \\
\text { transport. }\end{array}$ \\
$\begin{array}{l}\text { Limited consumer choice and poor } \\
\text { variety of services. }\end{array}$ & $\begin{array}{l}\text { providers. } \\
\text { Government-funded public transit. }\end{array}$ \\
Public and private partnership.
\end{tabular}

Source: Author's own research.

\footnotetext{
${ }^{3}$ https://www.mckinsey.com/business-functions/sustainability-and-resource-productivity/ourinsights/urban-mobility-at-a-tipping-point
} 
The individual-based mobility is facing changes due to the new business models in the sector. The following shifts can be noticed: 1) from private car ownership to car sharing; 2) from taxi services to e-haling (process of ordering a taxi or car via an on-demand application. App matches a client and a driver).

Concerning the group-based mobility, it is also facing new tendencies such as the shift from public transit to on-demand private shuttles (more convenient than public transport and cheaper than a taxi) and carpooling.

A shift towards new urban business strategies can provide such benefits as major savings in public budgets including health, environment or energy by providing a safer transport, less congestion and a higher rate of employment ${ }^{4}$.

\section{Mobility as a Service}

The concept of "transportation" based on a modal approach is evolving into the wider concept of mobility based on a service approach, for instance Mobility as a Service (MaaS). Nowadays, society gives preference to the customer experience more than to the vehicle ownership recognizing the use of various transport modes. For many people the ownership of a private car is no longer a primary objective for travelling, especially in urban areas. This conceptual change will completely modify the mobility offer, as well as the payment of mobility services (Kamargianni et al., 2016). The emerging travel services (such as car- and bike-sharing, ride-sharing, etc.) will have a positive impact on the urban mobility, as they permit to reduce the number of private cars and traffic congestion in dense urban areas (Wong et al., 2017). MaaS objectives are to put the users at the core of mobility services, offering them personalised mobility solutions based on their individual needs (Goodall et al., 2017).

MaaS has three dimensions that should take place when planning the innovation activities: The technological dimension: data sharing, interoperability, standardization as well as connectivity and built-in sensors of smart devices; The behavioural impact: the way travel and logistics patterns change (e.g. for older travellers) and what is the potential modal shift; The economic and policy dimension: including organizational and regulatory aspects. This might involve a change of roles of the different involved players.

Furthermore, according to Jittrapirom et al. (2017), a MaaS has the following core characteristics: the integration of transport modes, a tariff option, one platform, multiple actors, the use of technologies, personalisation and customisation.

\section{Integrated mobility}

Secondly, it is important to take into account the concept of integrated mobility. This approach enables connecting travellers from the trip origin to their final destination using all transportation modes through the integration of barrier-free planning, design, infrastructure, technology solutions and personalisation (Kamargianni et al., 2016). The concept behind integrated mobility is that passengers typically use more than one mode of transportation. Travelers have different trip needs and often switch modes to suit their travel needs.

The benefits that integrated mobility can provide are:

\footnotetext{
${ }^{4}$ Eurostat figure. European Commission webpage on mobility facts and figures: http://ec.europa.eu/transport/strategies/facts-and-figures/transport matters/index_en.htm
} 
- Combined mobility. Create a seamless travel experience for the door-to-door journey by integrating public and private transport modes in one single service, guided by an intermodal journey planner.

- One-stop-shop. Provide easier travelling by combining journey planning, mobile ticketing and fare collection in one single application.

- Personalized solutions. Every traveller has her/his own travel behaviour regarding the travel purpose, final destination and time. Each traveller needs the flexibility to choose and adapt her/his individual package (Stiglic et al. 2018).

A good example of integrated mobility can be a Google Trip Planner that enables to identify the best route and smarter decisions of traveling (Mahmoud et al., 2015).

The addition of alternative modes (transit, cycling and walking) to trip planning apps provide the traveller with information to compare travel times, identify different travel routes and better navigate through the transit network. Therefore, technology plays an important role in providing valuable information that allows travellers to make smarter travel choices and should be an essential part of integrated mobility.

\section{Shared Mobility}

The Swiss Mobility Academy 5 defines shared (or cooperative) mobility as follows: "Collaborative mobility focuses on sharing journeys, modes of transport, and infrastructure. In between collective and individual transport, new peer-to-peer based networks are emerging, boosting new types of individual mobility beyond private car ownership."

Shared mobility is part of the wider "collaborative economy" or "sharing economy" environment, defined in the European agenda for the collaborative economy as "[a variety of] innovative business models where activities are facilitated by collaborative platforms that create an open marketplace for the temporary usage of goods or services often provided by private individuals" ${ }^{\prime \prime}$. Service providers offer their goods, assets, or skills to a variety of users via a platform provided by intermediaries. "Sharing" has also become an urban mobility reality. Shared mobility prioritizes the importance of reaching destinations, often at a smaller individual and societal cost than by using a private vehicle. As shared mobility serves a greater proportion of local transportation needs, multivehicle households can begin reducing the number of cars they own while others may abandon ownership altogether, reducing future demand.

\section{Innovative Business Models in Mobility Sector}

It is now crucial to analyse what has been studied in the existing literature about the innovative business models in our sector of interest. Some researchers in the transport and mobility sector argue that the traditional organizational structure and Business model (BM) is no longer viable (Abdelkafi et al., 2013; Wells, 2013). Increasing challenges in the mobility sector such as market saturation, environmental issues (bad air conditions), congestion, accelerated urbanisation changing customers' demand and needs forcing the mobility organisation to change their BM in order to address these issues. Changing market

\footnotetext{
${ }^{5}$ Mobility Academy, 2014, Wocomoco flyer (http://bit.ly/2lnlwkq).

${ }^{6}$ European Commission, 2016, Communication A European agenda for the collaborative econ- omy http://bit.ly/2cFpEKq
} 
characteristics and fast evolving new technologies make LA to reorganize or even innovate their BM (Holweg, 2008).

Technological breakthrough also enables to enhance the improvements and technological advancements in many areas of transport and mobility, e.g. alternative power trains, digitalization, automotive software and hardware, connectivity and smartphone technologies that are further influencing the growth of innovative BM in the transport sector. Abdelkafi et al., (2013) argued that "Technology innovations and business model innovations are strongly linked to each other. A business model denoted the way companies can make money out of a technology. No matter how the technology is innovative and sophisticated, it will fail, if it is not possible for market players to make profits from it".

Following this statement, it can be derived that the emerging technological innovation of the transport industry should be accompanied by BM innovation.

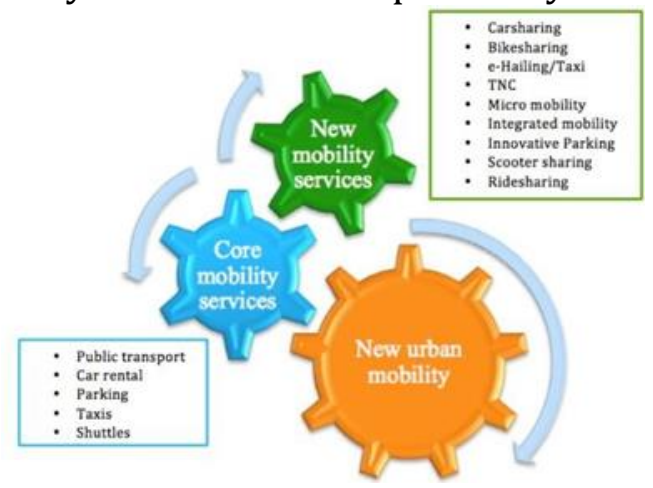

Figure 1. Technology Enhanced Urban Mobility Ecosystem

Source: Author's own research.

The new mobility paradigm is changing the urban mobility sector providing new business models and the ways transport authorities deliver services. New business models regard the above described MaaS, Sharing and Integrated mobility services. The next sections will introduce the innovative business models and partnerships representing current innovative mobility trends.

\section{Research Design and Methodology}

In order to answer our research question, a primary and a secondary data analysis were carried out to explore the economic viability and public acceptability of measures and to assess the success factors and barriers that may accelerate or inhibit the uptake to implement the innovative and sustainable urban mobility solutions in S-M cities.

The primary sources include a focus group of 5 people and informal conversations with city partners. These meetings helped to understand the urban mobility situation in these cities and to identify the most relevant mobility services.

The secondary data collection includes a triangulation of official governmental documents of EU and other countries, publications from peer-reviewed journals, media publications, web sites and books, as well as open source reports and papers, to guarantee greater validity to the investigation. 


\section{SUITS Project - Goals and Objectives}

Previously in this paper we described the issues concerning the current mobility scenario and some new trends and solutions that are becoming popular in urban environments thanks to the wave of digital transformation positively affecting this sector.

The overall aim of SUITS project is to support the S-M cities in the enhancement of their capacity to reduce congestion and air pollution, as well as to develop sustainable mobility in order to improve the urban mobility situation.

SUITS intends to achieve these goals by applying decision making support tools in developing innovative and sustainable financing, the implementation of schemes for innovative procurement, and providing innovative business models and partnerships.

Under SUITS Work Package 4 (WP) three Guidelines were developed in order to support local authorities in S-M cities to integrate new policies regarding sustainable transportation, including:

- Guidelines for applying Innovative and sustainable financing approaches.

- Guidelines for Innovative procurements.

- Guidelines for Developing bankable projects, new business models and partnerships.

The goal of these Guidelines is to provide an efficient decision-making tool that enables private and public mobility authorities/ stakeholders to improve the transport and mobility situation in their cities. The Municipality of Alba Iulia, Romania will test the ideas of three Guidelines.

After the finalization of all Work Packages, nine partner cities (Coventry City Council, Stuttgart, Erfurt, Palanga, Rome, Valencia, Torino, Alba Iulia, Kalamaria) will integrate the know-how delivered by the SUITS project including three Guidelines, the Pilot Application Report and the integrated tools. The project foresees also the training activities organized under different WP.

\section{Innovative Public Private Partnerships}

Some researchers sustain that societies are governed by a combination of governing efforts by different kinds of actors and entities, public as well as non-public (Kooiman, 2003; Kooiman, 2009).

Public-private partnerships (PPP) are well known to be powerful tools in carrying out transport and mobility projects. Public-private alliances are vital for the formulation and implementation of strategies in a mobility sector. Before introducing the Innovative Public Private Partnership (IPPP) it is necessary to present the PPP form explaining its benefits and challenges for mobility organisations.

\section{$P P P$}

Most EU member states and the European Commission regard the PPP as an important tool to attract additional financial resources for high priority investments such as transport. The European Commission gave the following definition of PPP by referring to "forms of cooperation between public authorities and businesses, with the aim of carrying out infrastructure projects or providing services to the public" (European Commission, 2005).

The public sector plays an important role in building the urban transport infrastructure. However, the resources needed are much more than what the public sector 
can provide and the public investment. Hence, they have to be supplemented by private sector investments. In order to address this gap, this Section is focusing on providing insights on the combination of public and private partnership and how this partnership can create a benefit for both urban mobility stakeholders.

The PPP forms an important part of the urban investment package (set of the presentation material and documents needed to obtain an investment). Many cities have PPP-specific departments to manage the role of the private sector in municipal service delivery, which tend to vary from city to city. PPPs can vary from basic service contracts with limited asset ownership, to mature fee-based build-operate-transfer (BOT) projects where the private sector has full responsibility for a project's operations and investment.

Among the benefits of using the PPP models, according to the $\mathrm{EC}^{7}$ are: acceleration of infrastructure provision, faster implementation, reduced entire life, better risk allocation, better incentives to perform, improved quality of service, generation of additional revenues, enhanced public management.

\section{$I P P P$}

IPPP is a new form of partnership where the main actors are public and private organisations and other type of organisations like civil society organizations (CSOs), nongovernmental organizations (NGO) or communities. These new forms of collaboration enable to identify the opportunities for the design and implementation of the long-term strategies for partnership. Each actor of the iPPPs has its important role in the alliance.

In the IPPPs, public organisations are defined as an important actor who not only has key roles of supervising, creating incentives and regulatory frameworks, but also developing new opportunities and governance mechanisms to enable the sustainable longlasting collaboration with the private sector and other forms of organization, in order to optimize outcomes, impact and sustainability.

The private sector has a significant role in the partnership. It contributes to bringing the investment and expertise in the alliance, having its business for-profit orientation.

Finally, other important actors in this type of partnership such as NGOs, CSOs or communities may bring their expertise and vision of transport and mobility sector.

The transport and mobility sector can benefit from this new form of partnership by having on board not only the professionals of the mobility sectors but also other important actors that may be more familiar with the transport sector issues and needs. The last ones could provide their attitude on the situation and help to develop more effective measures to address mobility issues. The logic of iPPPs is that the transport and mobility issues should be analysed jointly, rather than separately, by governments, public and private sector and communities.

\section{Innovative Business Models}

S-M-sized cities operate in the situation where the mobility sector faces a great competitiveness, a rapidly changing and uncertain economic environment that make local authorities (LA) to take complex and difficult business decisions. Transport and mobility organisations run their businesses in a digital era where new technologies innovate business models enabling to solve current mobility problems.

\footnotetext{
${ }^{7}$ http://ec.europa.eu/regional_policy/sources/docgener/guides/ppp_en.pdf
} 
Many factors should be taken into consideration while starting a new business such as mobility business environment, strategic partnerships, technological innovation, market tendencies, revenue streams etc. A well -elaborated business model will enable the transport and mobility organisations to obtain the funds for its innovation exploitation and a well-prepared feasibility study will prove the project's financial viability. This final section briefly introduces new mobility services and their business models (BM) that can be used as a fundamental tool to enable S-M-sized cities to create their mobility innovation strategies. The business model examples presented can contribute to the literature on this topic and be used as a cognitive model for the mobility stakeholders who want to deliver these kinds of services in their cities.

\section{Car on-demand}

On-demand mobility is the integration of ride-sharing and ride-hailing services with transit operations. Car on-demand is an innovative, user-focused approach which leverages emerging mobility services, integrated transit networks and operations, real-time data, connected travellers, and cooperative Intelligent Transportation Systems (ITS) to allow for a more traveller-centric, transportation system, providing improved mobility options to all travellers and users of the system in an efficient and safe manner.

\section{Micro Mobility}

Micro mobility refers to a brand new category of vehicles that can become an alternative to traditional modes of transportation. Several types of micro mobility vehicles exist: personal transportation solutions, such as scooters/E-scooters, E-bikes etc. and small electric cars with one or two seats, electric vehicles (EVs). The micro mobility vehicles industry is developing rapidly. Consumer preferences are changing and BM are striving to analyse and meet the new needs of the modern customers. For instance, the millennials generation' members are buying their first cars much later than previous generations. Small sized micro eclectic cars and personal transportation devices could be an attractive alternative to fossil fuel private cars.

\section{Car Sharing}

Car sharing is a service that provides customers with access to vehicles for short-term usually hourly use. The shared cars are distributed across a network of locations within a metropolitan area. Members can access the vehicles at any time with a reservation and are charged by time or by mile. Car sharing thus provides some of the benefits of a personal vehicle without the costs of owning a private one.

\section{Ride Sharing}

Traditional ride sharing includes carpooling (grouping of travellers into a privately owned vehicle, typically for commuting), vanpooling (sharing of a ride in a van by commuters traveling to/from a job centre) and real-time ridesharing services (matching of drivers and passengers based on destination, through a mobile app before the trip starts and through which the passenger pays a share of the trip cost).

Ridesharing essentially focuses on the issue of filling empty seats in vehicles, which helps better realize the vehicle occupancy potential and reduces the number of vehicles on 
the roadway. Ridesharing can be a powerful tool to address problems of congestion, emissions and fossil fuel dependency.

\section{Bike Sharing}

Bikes haring comes in multiple forms, including public, closed community and peer- to-peer systems. Bike sharing enables users to take short point-to-point trips using a fleet of public bikes distributed throughout a community. Peer-to-peer bike sharing allows users to rent or borrow bikes hourly or daily from individuals or bike rental shops. Bike sharing has the potential to play an important role in bridging some of the gaps in the existing transportation networks, as well as encouraging individuals to use multiple transportation modes. The ultimate goal of public bike sharing is to expand and integrate cycling into transportation systems, so that it can become a daily transportation mode for commuting.

\section{Parking}

In metropolitan areas, parking management influences drivers' search time and cost for parking spaces, parking revenue, and traffic congestion. The wide deployment of wireless parking meters with sensing and communications capabilities allows the parking authority to monitor the state of each parking space in real time and optimize the parking management. Innovations on this sector could prove to be very helpful especially in small and medium sized cities, where due to the absence of several different modes of transport, citizens rely almost entirely to their private cars for transportation. Thus, parking problems may appear more often on these cities.

\section{Public Transport}

Public transit encompasses publicly owned fleets of buses, trains, ferries, facilities etc., with fixed local routes and express services. It is a core of shared urban mobility. There is a great potential for transit agencies to integrate with or offer shared modes to increase access to transportation and lower costs. Both large technology companies and emerging app entrepreneurs are working to develop integration platforms that cross these modes.

\section{Integrated Mobility}

Integrated mobility is a technology-enabled strategic service to ensure that travellers have the most convenient possible transportation journey. Integrated mobility aims at connecting people and places.

\section{Conclusions and Future Research}

Cities are oriented to deliver sustainable solutions that can positively affect the lives of the world's population, improving the climate situation regarding the air pollution, reducing traffic congestion, developing a more secure transport and providing economic and social benefits. On the wave of the digital transformation of the society, promising urban service innovations that already exist still need to be scaled up to their full potential for small and medium cities to fully realize the benefits of sustainable urban development. A successful implementation of investment programs requires shifts in traditional business models that bring public and private interests into alignment. New approaches to developing sound 
project pipelines are needed to smooth and accelerate the early stage investment process where important knowledge, capacity and interest gaps can exist.

Finding new business models, like those mentioned in the present work, to accelerate and scale up investment in sustainable urban mobility will depend on answering the following key questions: what to invest in, how to pay for it, how to mobilize investment capital and how to structure implementation. To best answer these questions, the following key stakeholders need to develop sustainable solutions together: city decision makers, service providers and investors should build a shared understanding of the challenges and opportunities of different business model choices to ensure cities meet their service objectives; infrastructure and technology providers satisfy their bottom line; and financiers make a return on their investment. During the focus group meeting participants raised an issue that each city has its own mobility reality and new measures should be applied according to the local situations in the cities.

Despite this, the study can be useful for mobility stakeholders who want to apply the SUITS project findings and recommendations regarding new business models and partnership. A successful implementation of the guidelines will depend on the local governance and legislation, and the ability of public and private authorities to provide the resources for creation of new business opportunities.

The future research aims to provide an in-depth analysis of the new BM in the mobility sector and to analyse how the project cities managed to implement the proposed business models and what challenges they faced.

\section{References}

Abdelkafi, N., Makhotin, S., \& Posselt, T. (2013). Business model innovations for electric mobility - what can be learned from existing business model patterns? International Journal of Innovation Management, 17(01), 1340003.

Banister, D. (2008). The sustainable mobility paradigm. Transport policy, 15(2), 73-80.

Bell, D., \& Jayne, M. (2006). Small cities: Urban experience beyond the metropolis. Routledge.

Bellini, F., \& D'Ascenzo, F. (2018). L'evoluzione dei modelli di produzione nella logica della co-creazione di valore. In (a cura di) D'Ascenzo F., Bellini , F. (Eds.). Produzione, Logistica e Trasformazione Digitale (pp. 1-23). PADOVA: CEDAM, ISBN: 9788813366360.

Benevolo, C., Dameri, R. P., \& D’Auria, B. (2016). Smart Mobility in Smart City. In Torre, T., Braccini, A.M. ,Spinelli, R. (A c. Di). Empowering Organizations (pp. 13-28). Springer International Publishing.

Cohen, B. (2006). Urbanization in developing countries: Current trends, future projections, and key challenges for sustainability. Technology in society, 28(1-2), pp. 63-80

Dargay, J., Gately, D., \& Sommer, M. (2007). Vehicle ownership and income growth, worldwide: 1960-2030. The Energy Journal, 28(4), 143-170.

European Commission (2005). The Trans-European Transport Networks (TEN-T), 2005. European Commission (2017). European Urban Mobility, Policy context. Retrieved from https://ec.europa.eu/transport/sites/transport/files/2017-sustainable-urbanmobility- policy-context.pdf. 
Frumkin, H. (2002). Urban Sprawl and Public Health. Public Health Reports, 117(3), 201217. https://doi.org/10.1093/phr/117.3.201.

Goodall, W., Dovey, T., Bornstein, J., \& Bonthron, B. (2017). The rise of mobility as a service. Deloitte Rev, 20,. 112-129.

Holweg, M. (2008). The Evolution of Competition in the Automotive Industry. In Parry, G., Graves, A. (Eds.), Build To Order: The Road to the 5-Day Car ( pp. 13-34). https://doi.org/10.1007/978-1-84800-225-8_2.

Jittrapirom, P., Caiati, V., Feneri, A. M., Ebrahimigharehbaghi, S., González, M. J. A., \& Narayan, J. (2017). Mobility as a Service: a critical review of definitions, assessments of schemes, and key challenges. Urban Planning, 2(2), 13.

Kamargianni, M., Li, W., Matyas, M., \& Schäfer, A. (2016). A Critical Review of New Mobility Services for Urban Transport. Transportation Research Procedia, 14, 3294-3303. https://doi.org/10.1016/j.trpro.2016.05.277.

Kooiman, J. (2003). Governing as Governance. SAGE.

Kooiman, J., Jentoft, S. (2009). Meta-governance: values, norms and principles, and the making of hard choices. Public administration, 87(4), 818-836.

Mahmoud, M. S., Habib, K. M. N., \& Shalaby, A. (2015). Survey of Cross-Regional Intermodal Passenger Travel: Joint Revealed Preference-Stated Preference Survey Integrated with a Multimodal Trip Planner Tool. Transportation Research Record, 2526(1), 108118. https://doi.org/10.3141/2526-12.

Spickermann, A., Grienitz, V., \& Heiko, A. (2014). Heading towards a multimodal city of the future?: Multi-stakeholder scenarios for urban mobility. Technological Forecasting and Social Change, 89, 201-221.

Stiglic, M., Agatz, N., Savelsbergh, M., \& Gradisar, M. (2018). Enhancing urban mobility: Integrating ride-sharing and public transit. Computers \& Operations Research, 90, 1221.

Wells, P. (2013). Sustainable business models and the automotive industry: A commentary. IIMB Management Review, 25(4), 228-239.

Wong, Y. Z., Hensher, D. A., \& Mulley, C. (2018). Emerging transport technologies and the modal efficiency framework: A case for mobility as a service (MaaS) [Working Paper]. Retrieved from https://ses.library.usyd.edu.au/handle/2123/19100. 